\title{
Association of physical activity trajectories with major cardiovascular diseases in elderly people
}

\author{
Claudio Barbiellini Amidei ำ, ${ }^{1}$ Caterina Trevisan, ${ }^{2,3}$ Matilde Dotto, ${ }^{4}$ Eliana Ferroni, ${ }^{4}$ \\ Marianna Noale, ${ }^{5}$ Stefania Maggi, ${ }_{1}^{5}$ Maria Chiara Corti, ${ }^{4,6}$ Giovannella Baggio, ${ }^{2,7}$ \\ Ugo Fedeli, ${ }^{4}$ Giuseppe Sergi ${ }^{2}$
}

\begin{abstract}
- Additional supplemental material is published online only. To view, please visit the journal online (http://dx.doi. org/10.1136/heartjnl-2021320013)
\end{abstract}

'Department of Cardio-ThoracoVascular Sciences and Public Health, University of Padua,

Padova, Italy, Italy

2Department of Medicine,

Geriatrics Section, University of

Padua, Padua, Italy

${ }^{3}$ Department of Medical

Sciences, University of Ferrara,

Ferrara, Italy

${ }^{4}$ Epidemiological Department,

Azienda Zero, Padova, Italy

${ }^{5}$ Aging Branch, Institute of

Neuroscience, National Research

Council, Padova, Italy

${ }^{6}$ Azienda ULSS 6 Euganea,

Padova, Italy

${ }^{7}$ Internal Medicine Division,

Padua University Hospital,

Padua, Italy

\section{Correspondence to}

Dr Claudio Barbiellini Amidei,

University of Padua, Padova

35122, Italy;

claudioamidei@gmail.com

Received 29 July 2021

Accepted 18 November 2021

Published Online First

14 February 2022

\section{Linked}

- http://dx.doi.org/10.1136/ heartjnl-2021-320462

Check for updates

(c) Author(s) (or their employer(s)) 2022. No commercial re-use. See rights and permissions. Published by BMJ.

To cite: Barbiellini Amidei $\mathrm{C}$, Trevisan C, Dotto M, et al. Heart 2022;108:360-366.

\section{ABSTRACT \\ Introduction Cardiovascular benefits deriving from} physical activity are well known, but it is unclear whether physical activity trajectories in late life are associated with different risks of cardiovascular diseases.

Methods Progetto Veneto Anziani (Pro.V.A.) is a cohort study of 3099 Italians aged $\geq 65$ years with baseline assessment in 1995-1997 and follow-up visits at 4 and 7 years. Surveillance was extended to 2018 by linkage with hospital and mortality records. Prevalent and incident cardiovascular diseases (coronary heart disease, heart failure and stroke) were identified through clinical examination, questionnaire, or hospital records. Moderate to vigorous physical activity was considered as a time-varying variable. Physical activity trajectories were categorised as: stable-low, high-decreasing, lowincreasing and stable-high. Exposure was also assessed at $70,75,80$ and 85 years.

Results Overall, physical activity was associated with lower rates of incident cardiovascular diseases. A significant risk reduction was present among men and was stronger earlier in late life (70-75 years). Trajectories of stable-high physical activity were associated with a significantly lower risk of cardiovascular outcomes among men (HR $0.48,95 \% \mathrm{Cl} 0.27$ to 0.86 ) compared with those with stable-low trajectories ( $p$ for trend 0.002). No significant association was found with stroke. The greatest cardiovascular risk reduction was observed for $>20 \mathrm{~min} /$ day of physical activity, and was more marked at 70 years.

Conclusion Increasingly active trajectories of physical activity were associated with lower rates of cardiovascular diseases and overall mortality. Promoting at least $20 \mathrm{~min} /$ day of physical activity early in late life seems to provide the greatest cardiovascular benefits.

\section{INTRODUCTION}

Benefits deriving from physical activity in reducing cardiovascular risks have been well established. ${ }^{1-3}$ The World Health Organization (WHO) recommends at least $20 \mathrm{~min} /$ day of moderate to vigorous physical activity for older adults. ${ }^{4}$ The doseresponse association of cardiovascular outcomes with increasing duration of daily physical activity has shown a marked reduction in the incidence of any cardiovascular disease (CVD) ${ }^{56}$ including coronary heart disease (CHD), ${ }^{78}$ heart failure ${ }^{9} 10$ and stroke. ${ }^{5}$ These studies rarely focused on older adults, and few stratified risks based on sex. ${ }^{3}$ Evidence in the literature suggests a significant effect of physical activity in improving health in both men and women, but there are limits to the conclusions that can be drawn from these studies. ${ }^{3}$

Most studies have estimated health benefits based on a single measurement of physical activity, while a very relevant role seems to be played by trajectories of physical activity. ${ }^{11-13}$ The literature on this topic is scarce with contrasting results, ${ }^{14}$ but the findings suggest a reduction in the incidence of cardiovascular events with increasing levels of physical activity from mid-life to early old age. ${ }^{11}$ Few studies have examined the association of physical activity trajectories with specific cardiovascular outcomes, especially with regard to sex as well as age difference in elderly people. ${ }^{15}$

CVDs lead to 17.9 million deaths each year worldwide and are responsible for a very high burden of disease. ${ }^{16}$ Physical activity is considered to contribute to a reduction in the risk of CVD of $30-50 \% .{ }^{17}$ Given ageing populations and the public health relevance of CVDs, our objective is to examine how different trajectories of physical activity are associated with the incidence of cardiovascular events in older men and women.

\section{METHODS}

\section{Study cohort}

Progetto Veneto Anziani (Pro.V.A.) is a cohort study of 3099 Italians aged 65 years or older. Baseline assessments were carried out between 1995 and 1997, with two follow-up visits after 4 and 7 years. A thorough medical history and physical examination was performed by physicians at all visits along with biohumoral parameters, radiological examinations and the administration of validated scales, tests and questionnaires. Morbidity and mortality surveillance for the entire cohort was extended up to 31 December 2018 through linkage with hospital discharge records and mortality records. Other details concerning the Pro.V.A. study have been reported elsewhere. ${ }^{18}$

\section{Physical activity}

Data on physical activity were collected by means of a questionnaire at baseline and follow-ups. Information on the hours spent each week on specific activities allowed us to categorise physical activity as moderate to vigorous. ${ }^{19}$ Moderate physical activity included walking, playing games that require moderate physical exercise such as bowls, 
and fishing. Vigorous physical activity included gardening, gym work, biking, dancing and swimming. Hours of moderate and vigorous physical activity were then transformed into min/day, calculated as moderate +2 *vigorous physical activity. ${ }^{20}$ Participants were then defined as active or inactive if they engaged for $>20 \mathrm{~min} /$ day or $\leq 20 \mathrm{~min} /$ day, respectively, in these activities, according to WHO recommendations. ${ }^{4}$ Physical activity trajectories were identified when data were available from two or more visits. Four types of trajectories were defined: stable-low (inactive-inactive), high-decreasing (active-inactive), low-increasing (inactive-active) and stable-high (active-active). For sensitivity analysis, trajectories were also defined by all three available visits (stable trajectories).

\section{Outcome}

At baseline and follow-up assessments the prevalence of CVD was identified from medical history, physical examinations, electrocardiography and questionnaires to assess prevalent health conditions. Linkage with electronic health records (from January 1997 to December 2018) enabled the identification of all incident cases with a hospital diagnosis of CHD (ICD-9 codes 410-411, 414), heart failure (ICD-9 code 428) and stroke (ICD-9 codes $430-431,433-434,436)$. Incident CVD was defined as the date of the first event of CHD, heart failure or stroke.

\section{Covariates}

All analyses were stratified by sex and adjusted for a number of covariates assessed at baseline.

\section{Socioeconomic variables}

Education level (low (none, third grade, fifth grade), middle school (junior high, professional classes), high school or above (high school, pre-college, university degree)), number of household members (none, at least one) and year of birth categorised into 5 -year groups.

\section{Health behaviours}

Smoking (non-smoker, smoker, former smoker) and drinking habits (none or occasional alcohol consumption; $<7$ units of alcohol/week for women and $<14$ units/week for men; $\geq 7$ units/ week for women and $\geq 14$ units/week for men).

\section{Multimorbidity}

The number of chronic conditions was determined by clinical evaluations based on medical history, physical examination, medications and questionnaire-based assessments. For the purpose of this study we considered the following two continuous variables. (1) Number of non-cardiovascular comorbidities (treated as a continuous variable) including cancer, chronic obstructive pulmonary disease, asthma, hypoacusia, hypovisus, diabetes, chronic kidney disease (clinical diagnosis or glomerular filtration rate $<60 \mathrm{~mL} / \mathrm{min}$ ), anaemia, Parkinson's disease, dyslipidaemia, osteoporosis with or without femur fracture, discopathy, osteoarthrosis (clinically or radiographically assessed), orthostatic hypotension (measured), urinary incontinence and bowel incontinence. (2) Number of cardiovascular comorbidities (treated as a continuous variable, excluding the outcome of interest) including CHD, heart failure, stroke, angina, transient ischaemic attack, peripheral artery disease, atrial fibrillation or hypertension. For example, cardiovascular comorbidity adjustment for analyses on incident CHD included prevalent heart failure, stroke, angina, transient ischaemic attack and peripheral artery disease at baseline.

\section{Statistical analyses}

Differences in the distribution of baseline characteristics of the cohort, defined by continuous parameters, were assessed by means of generalised linear regression models and confirmed by t-tests. A $\chi^{2}$ test was used to assess differences in categorical variables. All descriptive analyses were stratified by sex and CVD status at the end of follow-up.

For each participant, follow-up ended at the first date between outcome, death or end of follow-up (31 December 2018). For CVD analyses we excluded all participants with prevalent CHD, heart failure or stroke at baseline $(n=433)$. When analysing CHD, heart failure and stroke, we only excluded participants with the outcome of interest as prevalent baseline condition $(\mathrm{n}=146, \mathrm{n}=256$ and $\mathrm{n}=103$, respectively). Cox regression models were used to estimate hazard ratios (HR) for all exposures of interest. Proportional hazards assumption was verified both by testing Schoenfeld residuals (main exposure and global model) and by visually verifying plotted Schoenfeld residuals. Reported HRs refer to the fully adjusted model that includes potential confounders for the tested association (socioeconomic variables, health behaviours and multimorbidity). Nelson-Aalen cumulative hazards, adjusted for socioeconomic variables, were plotted after using a smoothed robust locally weighted regression and smoothing scatterplots (Stata 15.0 lowess). ${ }^{21}$

The effect of physical activity on cardiovascular outcomes was analysed by three approaches:

1. Physical activity at baseline and follow-up assessments were entered into the Cox regression models as a time-varying variable. As sensitivity analysis, risks of cardiovascular outcomes based on physical activity at baseline were also assessed. This latter analysis was used to plot smoothed Nelson-Aalen cumulative hazards of incident CVD with physical activity assessed at 70, 75, 80 and 85 years (considering the closest visit within 5 years of the age of interest).

2. The association of CVD, CHD, heart failure and stroke with continuous minutes of moderate to vigorous physical activity at baseline was modelled using restricted cubic splines with four knots (adjusted for socioeconomic variables). This same analysis was performed by modelling the association of incident CVD with the minutes of physical activity at $70,75,80$ and 85 years.

3. Trajectories of physical activity started at baseline, and follow-up began after the second available point of observation (first or second follow-up visit) that defined the trajectory. The four types of trajectories that were identified (stable-low, high-decreasing, low-increasing and stable-high) were entered into the Cox regression models as a categorical variable, and as continuous to estimate the $\mathrm{p}$ for linear trend. CVD, CHD, heart failure and stroke were considered as separate outcomes without stratifying by sex. When evaluating the exposure of physical activity trajectories at 70,75, 80 and 85 years, only overall CVD was considered as an outcome.

All analyses were performed using Stata Version 15.0 (StataCorp).

\section{RESULTS}

The initial cohort was composed of 3099 adults aged 65 years or older. After excluding participants with missing data, 2754 participants remained (see online supplemental figure 1), of which 1398 were women (60.2\%), with no significant age differences between men and women at baseline (mean (SD) age 75.1 (7.0) years). During the follow-up (up to 31 December 2018) we observed 1037 incident cardiovascular events. Most 
Table 1 Characteristics of the cohort at baseline based on cardiovascular disease (CVD) status at the end of follow-up

\begin{tabular}{|c|c|c|c|c|c|c|}
\hline & \multicolumn{2}{|l|}{ CVD } & & \multicolumn{2}{|l|}{ No CVD } & \\
\hline & Male $(n=423)$ & Female $(n=614)$ & & Male $(n=500)$ & Female $(n=784)$ & \\
\hline Follow-up, years, mean (SD) & $8.1(5.6)$ & $9.5(6.2)$ & & $10.5(7.3)$ & $14.1(7.2)$ & \\
\hline \multicolumn{7}{|l|}{ Sociodemographic factors } \\
\hline Age, mean (SD) & $75.6(7.2)$ & $75.2(6.6)$ & $p=0.15$ & $75.2(7.3)$ & $74.6(7.0)$ & $p=0.34$ \\
\hline \multicolumn{7}{|l|}{ Education level (\%)* } \\
\hline Low or none & $118(27.9)$ & $354(57.7)$ & & $154(30.8)$ & $410(52.3)$ & \\
\hline Middle school & $277(65.5)$ & $235(38.3)$ & & $298(59.6)$ & $332(42.3)$ & \\
\hline High school or above & $28(6.6)$ & $25(4.1)$ & $p<0.0001$ & $48(9.6)$ & $42(5.4)$ & $\mathrm{p}<0.0001$ \\
\hline \multicolumn{7}{|l|}{ No of household members } \\
\hline 0 & $34(8.0)$ & $150(24.4)$ & & $46(9.2)$ & $187(23.9)$ & \\
\hline$\geq 1$ & $389(92.0)$ & $464(75.6)$ & $\mathrm{p}<0.0001$ & $454(90.8)$ & $597(76.1)$ & $\mathrm{p}<0.0001$ \\
\hline \multicolumn{7}{|l|}{ Health behaviours } \\
\hline \multicolumn{7}{|l|}{ Physical activity † } \\
\hline Inactive & $89(21.0)$ & $250(40.7)$ & & $114(22.8)$ & $334(42.6)$ & \\
\hline Active & $334(79.0)$ & $364(59.3)$ & $p<0.0001$ & $386(77.2)$ & $450(57.4)$ & $\mathrm{p}<0.0001$ \\
\hline \multicolumn{7}{|l|}{ Physical activity trajectories ¥ } \\
\hline Stable-low & $22(6.8)$ & $97(20.3)$ & & $14(4.7)$ & $104(18.5)$ & \\
\hline High-decreasing & $44(13.6)$ & $87(18.2)$ & & $34(11.4)$ & $69(12.3)$ & \\
\hline Low-increasing & $36(11.1)$ & 76 (15.9) & & $34(11.4)$ & $84(15.0)$ & \\
\hline Stable-high & $221(68.4)$ & $218(45.6)$ & $p<0.0001$ & $216(72.5)$ & $304(54.2)$ & $p<0.0001$ \\
\hline \multicolumn{7}{|l|}{ Alcohol (units/week) § } \\
\hline Teetotalers & $186(44.0)$ & $514(83.7)$ & & $234(46.8)$ & $635(81.0)$ & \\
\hline Moderate drinkers & $104(24.6)$ & $89(14.5)$ & & $118(23.6)$ & $131(16.7)$ & \\
\hline Heavy drinkers & $133(31.4)$ & $11(1.8)$ & $p<0.0001$ & $148(29.6)$ & $18(2.3)$ & $p<0.0001$ \\
\hline \multicolumn{7}{|l|}{ Smoking status (\%) } \\
\hline Never & $105(24.8)$ & $533(86.8)$ & & $104(20.8)$ & $675(86.1)$ & \\
\hline Former & $262(61.9)$ & $51(8.3)$ & & $288(57.6)$ & $73(9.3)$ & \\
\hline Current & $56(13.2)$ & $30(4.9)$ & $p<0.0001$ & $108(21.6)$ & $36(4.6)$ & $p<0.0001$ \\
\hline \multicolumn{7}{|l|}{ Clinical risk factors } \\
\hline BMI, mean (SD) & $26.0(3.6)$ & $25.8(4.2)$ & $\mathrm{p}=0.27$ & $25.4(3.5)$ & $25.4(4.3)$ & $\mathrm{p}=0.12$ \\
\hline \multicolumn{7}{|l|}{ Multimorbidity } \\
\hline 0 & $26(6.2)$ & $25(4.1)$ & & $41(8.2)$ & $31(4.0)$ & \\
\hline 1 & $86(20.3)$ & $63(10.3)$ & & $104(20.8)$ & $83(10.6)$ & \\
\hline 2 & $87(20.6)$ & $104(16.9)$ & & $125(25.0)$ & $141(18.0)$ & \\
\hline 3 & $102(24.1)$ & $131(21.3)$ & & $94(18.8)$ & $172(21.9)$ & \\
\hline$\geq 4$ & $122(28.8)$ & $291(47.4)$ & $p<0.0001$ & $136(27.2)$ & $357(45.5)$ & $p<0.0001$ \\
\hline \multicolumn{7}{|c|}{ Prevalent cardiovascular comorbidity } \\
\hline 0 & $61(14.4)$ & $92(15.0)$ & & $86(17.2)$ & $137(17.5)$ & \\
\hline 1 & $362(85.6)$ & $522(85.0)$ & $\mathrm{p}=0.80$ & $414(82.8)$ & $647(82.5)$ & $\mathrm{p}=0.90$ \\
\hline
\end{tabular}

Numbers in the table refer to participants included in the analyses on incident CVD ( $\mathrm{n}=2321)$.

*Low or none (none, third grade or fifth grade), middle school (junior high, professional classes), high school or above (high school, pre-college or university degree).

tActive defined as $>20$ min of moderate to vigorous physical activity per day and inactive as $\leq 20$ min.

¥Numbers were defined regardless of prevalent CVD cases at the second available visit that defined the trajectory (only referred to participants with at least 2 visits).

$\S$ Teetotalers: none or occasional alcohol consumption; moderate drinkers: $<7$ units of alcohol/week for women and $<14$ units/week for men; heavy drinkers: $\geq 7$ units/week for women and $\geq 14$ units/week for men.

ๆAngina, transient ischaemic attack, peripheral artery disease, atrial fibrillation or hypertension.

participants had middle or low education, with a higher education level among men. Men were more likely to have at least one household member living with them at baseline. Most participants had stable active physical activity trajectories, which was more common in men, regardless of CVD status at the end of follow-up. Smoking and alcohol consumption were also more frequent among men than women, with no relevant differences in body mass index (table 1). At baseline, women were more likely to have more than four comorbid conditions and had an especially higher prevalence of osteoarthrosis, osteoporosis and chronic kidney disease, while chronic obstructive pulmonary disease and diabetes were more common among men (see online supplemental table 1).

Over the entire follow-up period the rates of incident CHD and heart failure were lower for both active men and women (table 2 and online supplemental table 2). Physical activity was associated with a significantly lower risk of CVD (fully adjusted HR $0.74,95 \%$ CI 0.58 to 0.94 ), CHD (HR 0.66, 95\% CI 0.50 to 0.87 ) and heart failure (HR $0.72,95 \%$ CI 0.53 to 0.98 ) in men (table 2). Similar results were observed when considering exclusively exposure at baseline (online supplemental table 2). Physical activity was associated with a significantly reduced risk 
Table 2 Association between physical activity* and incident CVD, CHD, heart failure and stroke, stratified by sex

\begin{tabular}{|c|c|c|c|c|c|c|c|c|}
\hline \multirow[b]{2}{*}{ Follow-up } & \multicolumn{4}{|l|}{ Male } & \multicolumn{4}{|l|}{ Female } \\
\hline & $\begin{array}{l}\text { Cases/ } \\
\text { total }\end{array}$ & $\begin{array}{l}\text { Rate/1000 } \\
\text { person-years }\end{array}$ & $\begin{array}{l}\text { Model 1† } \\
\text { HR }(95 \% \mathrm{Cl})\end{array}$ & $\begin{array}{l}\text { Fully adjusted } ¥ \text { model } \\
\text { HR }(95 \% \mathrm{Cl})\end{array}$ & $\begin{array}{l}\text { Cases/ } \\
\text { total }\end{array}$ & $\begin{array}{l}\text { Rate/1000 } \\
\text { person-years }\end{array}$ & $\begin{array}{l}\text { Model 1† } \\
\text { HR }(95 \% \mathrm{Cl})\end{array}$ & $\begin{array}{l}\text { Fully adjusted } ¥ \text { model } \\
\text { HR }(95 \% \mathrm{CI})\end{array}$ \\
\hline CVD & \multicolumn{4}{|c|}{ Mean (SD) follow-up 9.4 (6.7) years } & \multicolumn{4}{|c|}{ Mean (SD) follow-up 9.8 (6.9) years } \\
\hline Inactive & $89 / 203$ & 61.30 & Ref & Ref & $250 / 584$ & 40.72 & Ref & Ref \\
\hline Active & $334 / 720$ & 46.53 & 0.75 (0.59 to 0.95$)$ & $0.74(0.58$ to 0.94$)$ & $364 / 814$ & 33.74 & $0.95(0.80$ to 1.12$)$ & $1.00(0.85$ to 1.19$)$ \\
\hline CHD & \multicolumn{4}{|c|}{ Mean (SD) follow-up 9.6 (6.9) years } & \multicolumn{4}{|c|}{ Mean (SD) follow-up 8.9 (5.7) years } \\
\hline Inactive & $77 / 263$ & 42.28 & Ref & Ref & $182 / 689$ & 25.34 & Ref & Ref \\
\hline Active & $209 / 788$ & 25.33 & $0.65(0.49$ to 0.85$)$ & $0.66(0.50$ to 0.87$)$ & $208 / 868$ & 17.20 & 0.84 (0.68 to 1.03$)$ & $0.92(0.75$ to 1.14$)$ \\
\hline Heart failure & \multicolumn{4}{|c|}{ Mean (SD) follow-up 10.2 (6.8) years } & \multicolumn{4}{|c|}{ Mean (SD) follow-up 13.1 (6.9) years } \\
\hline Inactive & $58 / 227$ & 32.14 & Ref & Ref & $178 / 635$ & 24.58 & Ref & Ref \\
\hline Active & $221 / 797$ & 25.55 & $0.74(0.55$ to 1.00$)$ & $0.72(0.53$ to 0.98$)$ & $247 / 839$ & 20.40 & 0.91 (0.75 to 1.11$)$ & $1.02(0.83$ to 1.24$)$ \\
\hline Stroke & \multicolumn{4}{|c|}{ Mean (SD) follow-up 10.0 (6.8) years } & \multicolumn{4}{|c|}{ Mean (SD) follow-up $12.9(6.9)$ years } \\
\hline Inactive & $32 / 260$ & 15.85 & Ref & Ref & $101 / 701$ & 12.97 & Ref & Ref \\
\hline Active & $138 / 818$ & 15.78 & $1.02(0.69$ to 1.50$)$ & 1.02 (0.69 to 1.52$)$ & $149 / 872$ & 11.96 & $1.05(0.81$ to 1.36$)$ & $1.14(0.87$ to 1.48$)$ \\
\hline
\end{tabular}

Physical activity was entered in the model as a time-varying variable.

*Physical activity (moderate to vigorous physical activity) categorised as inactive $\leq 20 \mathrm{~min} /$ day and active $>20 \mathrm{~min} /$ day.

tModel 1: Adjusted for sociodemographic variables (sex, education and number of members living in the same household), stratified by categorised year of birth

łFully adjusted model: Model 1 further adjusted by health behaviours (smoking and alcohol consumption), number of chronic comorbid conditions (cancer, chronic obstructive pulmonary disease, asthma, hypoacusia hypovisus, diabetes, chronic kidney disease, anaemia, Parkinson's disease, dyslipidaemia, osteoporosis with or without femur fracture, congenital mental retardation, osteoarthrosis, discopathy, orthostatic hypotension, urinary incontinence and bowel incontinence) and comorbid cardiovascular diseases (CHD, heart failure, stroke, angina, transient ischaemic attack, atrial fibrillation, hypertension and peripheral artery disease with the exclusion of the outcome of interest) at baseline, and stratified by categorised year of birth.

CHD, coronary heart disease; CVD, cardiovascular disease.

of overall mortality in both men and women (HR 0.72, 95\% CI 0.62 to 0.84 and HR $0.81,95 \%$ CI 0.72 to 0.92 , respectively), as shown in online supplemental table 3 . With regard to the association of physical activity at different ages with the risk of any cardiovascular outcome, we found significantly reduced risks among men, only at age 70 (HR $0.58,95 \%$ CI 0.39 to 0.84 ) and limit to significance at age 75 (HR $0.74,95 \%$ CI 0.52 to 1.05 ), as shown in online supplemental figure 2. Physical activity was not associated with a reduced risk of stroke in any of the analyses. When including prevalent cases at baseline, the results did not vary (data not shown).
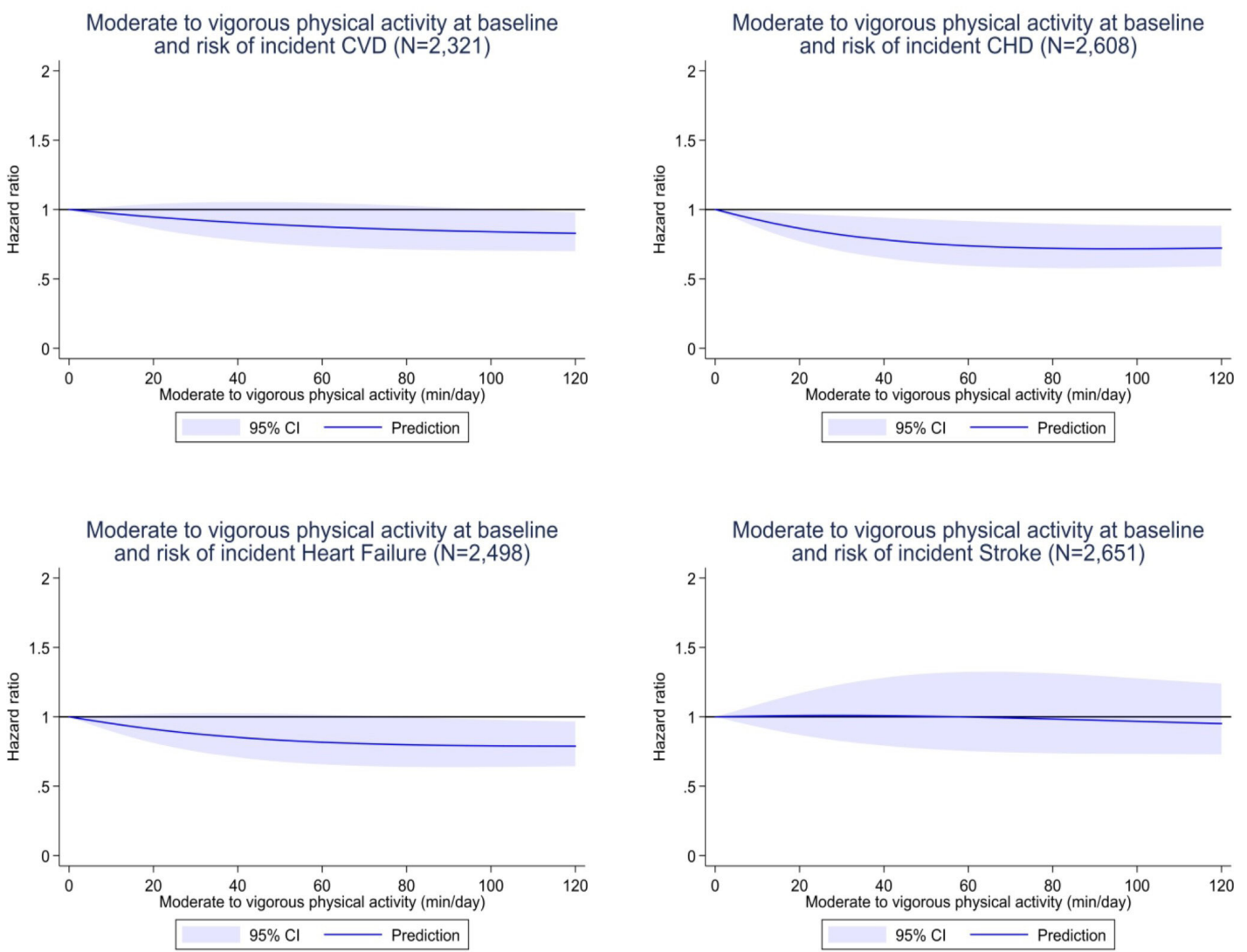

Figure 1 Association between moderate to vigorous physical activity (min/day) at baseline and incident cardiovascular disease (CVD), coronary heart disease (CHD), heart failure and stroke. Physical activity (PA) was considered as (moderate PA min/day)+2*(vigorous PA min/day). All splines were adjusted for sociodemographic variables and stratified by categorised year of birth. 
Table 3 Association between physical activity* trajectories from baseline with incident CVD, CHD, heart failure and stroke, stratified by sex

\begin{tabular}{|c|c|c|c|c|c|c|}
\hline \multirow[b]{2}{*}{ Follow-up } & \multicolumn{3}{|l|}{ Male } & \multicolumn{3}{|l|}{ Female } \\
\hline & $\begin{array}{l}\text { Cases/ } \\
\text { total }\end{array}$ & $\begin{array}{l}\text { Rate/1000 person- } \\
\text { years }\end{array}$ & $\begin{array}{l}\text { Fully adjustedt model } \\
\text { HR }(95 \% \mathrm{Cl})\end{array}$ & $\begin{array}{l}\text { Cases/ } \\
\text { total }\end{array}$ & $\begin{array}{l}\text { Rate/1000 person- } \\
\text { years }\end{array}$ & $\begin{array}{l}\text { Fully adjustedt model } \\
\text { HR }(95 \% \mathrm{Cl})\end{array}$ \\
\hline \multicolumn{7}{|c|}{ Any cardiovascular disease } \\
\hline & \multicolumn{3}{|c|}{ Mean (SD) follow-up from baseline 8.1 (5.4) years } & \multicolumn{3}{|c|}{ Mean (SD) follow-up from baseline $10.2(5.4)$ years } \\
\hline Stable-low & $13 / 27$ & 87.88 & Ref & $80 / 184$ & 48.12 & Ref \\
\hline High-decreasing & $31 / 65$ & 85.71 & $0.71(0.37$ to 1.39$)$ & $60 / 129$ & 55.04 & $1.28(0.91$ to 1.80$)$ \\
\hline Low-increasing & $26 / 60$ & 57.38 & 0.65 (0.33 to 1.29$)$ & $59 / 143$ & 40.04 & 0.94 (0.67 to 1.32$)$ \\
\hline Stable-high & 179/395 & 51.54 & $0.48(0.27$ to 0.86$)$ & 193/497 & 35.15 & 0.92 (0.70 to 1.21$)$ \\
\hline$p$ for trend & & & 0.002 & & & 0.239 \\
\hline \multicolumn{7}{|c|}{ Coronary heart disease } \\
\hline & \multicolumn{3}{|c|}{ Mean (SD) follow-up from baseline 8.6 (5.4) years } & \multicolumn{3}{|c|}{ Mean (SD) follow-up from baseline 10.7 (5.3) years } \\
\hline Stable-low & $13 / 37$ & 62.22 & Ref & $51 / 209$ & 26.39 & Ref \\
\hline High-decreasing & $20 / 77$ & 44.68 & 0.55 (0.27 to 1.12$)$ & $32 / 149$ & 23.54 & 0.99 (0.63 to 1.56$)$ \\
\hline Low-increasing & $15 / 66$ & 27.52 & $0.52(0.24$ to 1.10$)$ & $25 / 156$ & 14.62 & $0.68(0.42$ to 1.11$)$ \\
\hline Stable-high & $102 / 434$ & 24.91 & $0.40(0.22$ to 0.73$)$ & $96 / 527$ & 15.63 & 0.81 (0.56 to 1.17$)$ \\
\hline$p$ for trend & & & 0.005 & & & 0.185 \\
\hline \multicolumn{7}{|l|}{ Heart failure } \\
\hline & \multicolumn{3}{|c|}{ Mean (SD) follow-up from baseline 8.8 (5.3) years } & \multicolumn{3}{|c|}{ Mean (SD) follow-up from baseline $10.8(5.2)$ years } \\
\hline Stable-low & $9 / 33$ & 43.09 & Ref & $61 / 202$ & 31.36 & Ref \\
\hline High-decreasing & $24 / 86$ & 44.40 & $0.77(0.35$ to 1.69$)$ & $53 / 149$ & 39.44 & 1.41 (0.97 to 2.04$)$ \\
\hline Low-increasing & $23 / 70$ & 39.24 & 1.01 (0.46 to 2.20$)$ & $41 / 163$ & 22.40 & $0.82(0.55$ to 1.23$)$ \\
\hline Stable-high & $127 / 449$ & 29.59 & 0.60 (0.30 to 1.19$)$ & $138 / 529$ & 22.28 & 0.90 (0.66 to 1.24$)$ \\
\hline$p$ for trend & & & 0.038 & & & 0.175 \\
\hline \multicolumn{7}{|l|}{ Stroke } \\
\hline & \multicolumn{3}{|c|}{ Mean (SD) follow-up from baseline 8.6 (5.4) years } & \multicolumn{3}{|c|}{ Mean (SD) follow-up from baseline 10.6 (5.3) years } \\
\hline Stable-low & $7 / 44$ & 29.67 & Ref & $35 / 227$ & 16.60 & Ref \\
\hline High-decreasing & $10 / 85$ & 19.35 & $0.67(0.25$ to 1.78$)$ & $31 / 162$ & 21.61 & 1.34 (0.82 to 2.20$)$ \\
\hline Low-increasing & $11 / 76$ & 16.93 & $0.54(0.20$ to 1.43$)$ & $27 / 172$ & 14.58 & 0.99 (0.59 to 1.66$)$ \\
\hline Stable-high & $83 / 455$ & 19.43 & $0.63(0.28$ to 1.40$)$ & $79 / 536$ & 12.57 & 0.95 (0.62 to 1.44$)$ \\
\hline $\mathrm{p}$ for trend & & & 0.464 & & & 0.494 \\
\hline
\end{tabular}

*Physical activity (moderate and vigorous physical activity) categorised as inactive $\leq 20$ min day and active $>20$ min day (participants with at least two visits).

tFully adjusted model: adjusted for sociodemographic variables (sex, education and number of members living in the same household), health behaviours (smoking and alcohol consumption), number of chronic comorbid conditions (cancer, chronic obstructive pulmonary disease, asthma, hypoacusia, hypovisus, diabetes, chronic kidney disease, anaemia, Parkinson's disease, dyslipidaemia, osteoporosis with or without femur fracture, congenital mental retardation, osteoarthrosis, discopathy, orthostatic hypotension, urinary incontinence and bowel incontinence) and comorbid cardiovascular diseases (CHD, heart failure, stroke, angina, transient ischaemic attack, atrial fibrillation, hypertension and peripheral artery disease, with the exclusion of the outcome of interest) at baseline, and stratified by categorised year of birth.

CHD, coronary heart disease; CVD, cardiovascular disease.

When analysing the dose-response association of continuous minutes of weighted moderate to vigorous physical activity per day at baseline, we observed a reduction in the risk of incident CHD and heart failure which was specially marked between 20 and 40 min of physical activity per day, as shown in figure 1, with a J-shaped association for CHD ( $\mathrm{p}$ for linearity $=0.01$ indicating a non-linear spline). The risk reduction of any incident cardiovascular event associated with at least $20 \mathrm{~min}$ of physical activity per day was most marked at 70 years, and only moderately reduced at 75 years, while no significant correlation could be observed at 80 and 85 years (see online supplemental figure 3). When stratifying by sex, this association was more pronounced in men and only for CHD and heart failure (online supplemental figure 4).

Trajectories of physical activity, categorised by increasingly active status over time (stable-low, high-decreasing, lowincreasing and stable-high), were associated with a lower risk of incident CHD and heart failure among men ( $\mathrm{p}$ for trend 0.005 and 0.038 , respectively), as shown in table 3 and online supplemental figure 5. Although risk reduction was non-significant among more physically active women, the rates of incident CHD and heart failure were always lower for participants with low-increasing and stable-high levels of physical activity, as opposed to high-decreasing and stable-low physical activity. When these trajectories were observed at different ages from baseline, a trend of significantly reduced risks of any major incident cardiovascular event was found among men at 70 and 75 years ( $\mathrm{p}$ for trend 0.002 and 0.025 , respectively), as shown in online supplemental table 4. Among women at different ages, the association of increasing levels of physical activity trajectories with any cardiovascular outcome did not reach statistical significance, while risks for overall mortality were significantly reduced (see online supplemental table 3 and online supplemental table 4).

\section{DISCUSSION}

Cardiovascular benefits deriving from physical activity among adults are well established. ${ }^{1-3}$ In the present study, increasing levels of physical activity as well as the persistence of an active lifestyle over time seem to be associated with a reduced risk of incident CVDs in older age. Incidence rates of cardiovascular events were always lower among active men and women, as were overall mortality risks, similar to that reported in previous studies. $^{22}$ The greatest risk reduction was observed for incident CHD and heart failure. When stratifying by age, we found that the association between physical activity and cardiovascular risk was significantly reduced among participants aged 70 years, and with marginal significance at 75 years, while no association was found among the oldest age groups, suggesting greater cardiovascular benefits might be achieved by improving physical activity earlier in late life. 
The dose-response association of baseline physical activity with overall incident CVD and heart failure was linear, as previously reported in the literature, ${ }^{23}$ while the risk of CHD showed a J-shaped association. Previous studies also reported a curvilinear dose-response relationship of physical activity with incident CHD. ${ }^{7}$ The lowest effective dose of physical activity seems to be achieved with at least 20 min of physical activity per day, ${ }^{724}$ similar to that observed in our study. A clear dose-response association of incident CVD with minutes per day of physical activity was observed only at around 70 years, with a less marked reduction among older participants. Longer exposure to moderate and vigorous physical activity seems to be more effective in reducing cardiovascular risks early in late life.

Increasing levels of physical activity trajectories were also associated with a lower incidence of CVD, suggesting that improving and maintaining an active status even in advanced age should be recommended. In fact, compared with participants with stable-low trajectories, all other trajectories-and especially stable-high trajectories-had lower rates of incident CVD. Moreover, the results from this study suggest that the earlier people engage in physical activity, the greater reduction in incident CVD they may achieve. Conversely, the absence of any association with stroke has also been observed in a previous study. ${ }^{11}$ Despite the fact that stroke shares numerous risk factors with CVDs, including physical activity, an active lifestyle among older people could increase the risk for specific types of stroke such as haemorrhagic stroke deriving from vascular traumas, and results from previous literature have in fact been inconsistent. ${ }^{525}$

Women doing more physical activity had consistently lower incidence rates of almost all cardiovascular outcomes despite the fact that the risk reduction did not reach statistical significance, but when considering overall mortality, risks were significantly reduced. Although the magnitude of the studied associations was greater among men, the risks of CHD and heart failure were also moderately reduced after longer daily exposure to physical activity in women, as previously reported in the literature. ${ }^{3}$ However, despite the fact that sex-based differences of physical activity on cardiovascular health have been previously reported,${ }^{26}$ there is strong evidence in support of a positive effect of physical activity on cardiovascular risk reduction in both men and women. 2728

Mechanisms underlying the beneficial effects of physical activity on cardiovascular health are well known and can be attributed to numerous biological modifications. These include enhanced mitochondrial function, restored and improved vascular conditions and the release of myokines from skeletal muscle that enhance or preserve cardiovascular function. ${ }^{29}$ These modifications are likely to be more effective when the overall cardiovascular status has not been compromised by a long-lasting sedentary lifestyle. Moreover, the fact we found a significant association of physical activity with cardiovascular outcomes only early in late life could be attributed to the need for a longer exposure to an active lifestyle in order to modify cardiovascular risk profiles. ${ }^{25} 30$

\section{Strengths and limitations}

The main strengths of this study include detailed physical examinations at all visits and the long follow-up of a cohort of elderly participants which allowed us to assess outcomes from age 65 or above to death. Furthermore, to the best of our knowledge, the association between different trajectories of physical activity at specific ages in elderly people have not been previously analysed. Another strength is the stratification of all main analyses by sex, considering men and women are characterised by different cardiovascular risk profiles. Consistency of results across all sensitivity analyses further suggests the robustness of the main results.

Limitations to this study include the possibility of recall bias that could be responsible for an incorrect assessment of previous nonsevere cardiovascular conditions such as transient ischaemic attack and angina, especially among very old participants. Nevertheless, given the thorough physical examination, this bias is unlikely to concern severe cardiovascular event. Moreover, we did not have data on physical activity during mid-life that could have influenced the cardiovascular risk profiles in late life. Furthermore, physical activity was questionnaire-based with assessments about 3 years apart, and was not recorded by an accelerometer which could have ensured an objective measurement of the exposure. Despite the fact that most participants were physically active and men were more likely to be physically active than women, in the main analyses, all trajectories in both sexes were sufficiently represented. Another limit was the low number of participants in certain strata (eg, at 85 years) and a relatively low number of stroke events which did not allow further stratification for specific types of stroke (ie, ischaemic and haemorrhagic).

\section{CONCLUSION}

Physical activity among older people was associated with a reduced risk of CHD and heart failure, especially early in late life. Lower incidence rates of CVD were found among both physically active men and women. No significant association between physical activity and stroke was observed. Physical activity and increasingly active trajectories of physical activity were also associated with a significantly lower mortality risk in both men and women. These results suggest that public health policies should be targeted at promoting or beginning physical activity in mid- and early late life, given a probable greater effectiveness in reducing cardiovascular risks. At least 20 minutes of

Key messages

What is already known about this subject?

- Cardiovascular benefits deriving from physical activity are well known. Nevertheless, the dose-response relationship of physical activity with CVD among elderly people has not been examined. It is also unclear whether physical activity trajectories in late life are associated with different cardiovascular risk profiles.

\section{What does this study add?}

- In this 20-year follow-up cohort study of elderly people, the greatest benefit in cardiovascular risk reduction was obtained with at least $20 \mathrm{~min} /$ day of moderate to vigorous physical activity at around 70 years. Increasing levels of physical activity were associated with a reduced risk of cardiovascular outcomes early rather than late in late life (70 and 75 years). This was mostly attributable to a lower incidence of CHD and heart failure.

\section{How might this impact on clinical practice?}

- These results suggest that public health policies should promote physical activity in mid and early late life, rather than late in late life, given a probable greater effectiveness in reducing cardiovascular risks. At least $20 \mathrm{~min}$ of moderate or vigorous physical activity per day should be recommended to maximise cardiovascular benefits. 
moderate to vigorous physical activity per day should be recommended to achieve the greatest cardiovascular benefits.

Acknowledgements The authors would like to thank all the interviewers (nurses and physicians) who conducted the Pro.V.A. study and all the participants from Camposampiero and Rovigo who took part in it.

Contributors CBA conceived the study, performed the analyses, interpreted the results and wrote the manuscript, and is guarantor of this paper. CT contributed to the study design, revised the study, interpreted the results, revised the manuscript and provided the data. MD contributed to the study design, revised the study, interpreted the results and provided the data. EF contributed to the study design, revised the study, interpreted the results and revised the manuscript. MN revised the study, contributed to the statistical analyses, interpreted the results and revised the manuscript. SM revised the study, interpreted the results and revised the manuscript. MCC revised the study, revised the manuscript and provided the data. GB revised the study, interpreted the results and revised the manuscript. UF contributed to the study design, revised the study, contributed to the statistical analyses, interpreted the results, revised the manuscript and provided the data. GS revised the study, revised the manuscript and provided the data. All authors read and approved the final version of the manuscript.

Funding The Pro.V.A. study was supported by the Fondazione Cassa di Risparmio di Padova e Rovigo; the University of Padova (Population Ageing - Economics, Health, Retirement and the Welfare State - POPA_EHR); the Veneto Regional Local Health and Social Care Units 15 and 18 (Azienda Unità Locale Socio Sanitaria 15 and 18); and the Veneto Regional Authority (Ricerca Sanitaria Finalizzata n.156/03 and Ricerca Sanitaria Finalizzata RSF-2017-00000533).

Disclaimer The sponsor had no role in the design, methods, subject recruitment, data collections, analysis and preparation of the paper.

Competing interests None declared.

Patient consent for publication Not applicable.

Ethics approval This study involves human participants and was approved by the University of Padua, Local Health Units 15 and 18 of the Veneto Region, and the Provinces of Padua and Rovigo, and participants gave informed consent before taking part in the study.

Provenance and peer review Not commissioned; externally peer reviewed.

Data availability statement No data are available. The data we used for our study cannot be shared publicly. A researcher interested in gaining access to the relevant data should request them from the Regional Epidemiological Service of Veneto Region at ser@azero.veneto.it.

Supplemental material This content has been supplied by the author(s). It has not been vetted by BMJ Publishing Group Limited (BMJ) and may not have been peer-reviewed. Any opinions or recommendations discussed are solely those of the author(s) and are not endorsed by BMJ. BMJ disclaims all liability and responsibility arising from any reliance placed on the content. Where the content includes any translated material, BMJ does not warrant the accuracy and reliability of the translations (including but not limited to local regulations, clinical guidelines, terminology, drug names and drug dosages), and is not responsible for any error and/or omissions arising from translation and adaptation or otherwise.

\section{ORCID iD}

Claudio Barbiellini Amidei http://orcid.org/0000-0002-7792-3781

\section{REFERENCES}

1 Lear SA, Hu W, Rangarajan S, et al. The effect of physical activity on mortality and cardiovascular disease in 130000 people from 17 high-income, middle-income, and low-income countries: the PURE study. Lancet 2017;390:2643-54.

2 Lee J-Y, Ryu S, Cheong E, et al. Association of physical activity and inflammation with all-cause, cardiovascular-related, and cancer-related mortality. Mayo Clin Proc 2016:91:1706-16.

3 Shiroma EJ, Lee I-M, I-Min L. Physical activity and cardiovascular health. Circulation 2010:122:743-52.

4 Organisation Mondiale de la Santé. Global recommendations on physical activity for health. WHO, 2010.
5 Kubota Y, Evenson KR, Maclehose RF, et al. Physical activity and lifetime risk of cardiovascular disease and cancer. Med Sci Sports Exerc 2017;49:1599-605.

6 Powell KE, King AC, Buchner DM, et al. The scientific foundation for the physical activity guidelines for Americans, 2nd edition. J Phys Act Health. 2018:1-11.

7 Geidl W, Schlesinger S, Mino E, et al. Dose-response relationship between physical activity and mortality in adults with noncommunicable diseases: a systematic review and meta-analysis of prospective observational studies. Int J Behav Nutr Phys Act 2020;17:109.

8 Sattelmair J, Pertman J, Ding EL, et al. Dose response between physical activity and risk of coronary heart disease: a meta-analysis. Circulation 2011:124:789-95.

9 Florido R, Kwak L, Lazo M, et al. Six-year changes in physical activity and the risk of incident heart failure: ARIC study. Circulation 2018:137:2142-51.

10 Pandey A, Garg S, Khunger M, et al. Dose-response relationship between physical activity and risk of heart failure: a meta-analysis. Circulation 2015;132:1786-94.

11 Aggio D, Papachristou E, Papacosta O, et al. Trajectories of physical activity from midlife to old age and associations with subsequent cardiovascular disease and allcause mortality. J Epidemiol Community Health 2020;74:130-6.

12 Sanchez-Sanchez JL, Izquierdo M, Carnicero-Carreño JA, et al. Physical activity trajectories, mortality, hospitalization, and disability in the Toledo study of healthy aging. J Cachexia Sarcopenia Muscle 2020;11:1007-17.

13 Aggio D, Papachristou E, Papacosta 0, et al. Association between 20-year trajectories of nonoccupational physical activity from midlife to old age and biomarkers of cardiovascular disease: a 20-year longitudinal study of British men. Am J Epidemiol 2018; 187:2315-23

14 Laddu DR, Rana JS, Murillo R, et al. 25-Year physical activity trajectories and development of subclinical coronary artery disease as measured by coronary artery calcium: the Coronary Artery Risk Development in young adults (CARDIA) study. Mayo Clin Proc 2017:92:1660-70.

15 Wu S, An S, Li W, et al. Association of trajectory of cardiovascular health score and incident cardiovascular disease. JAMA Netw Open 2019;2:e194758.

16 Yazdanyar A, Newman AB. The burden of cardiovascular disease in the elderly: morbidity, mortality, and costs. Clin Geriatr Med 2009:25:563-77.

17 Mora S, Cook N, Buring JE, et al. Physical activity and reduced risk of cardiovascular events: potential mediating mechanisms. Circulation 2007;116:2110-8

18 Corti M-C, Guralnik JM, Sartori L, et al. The effect of cardiovascular and osteoarticular diseases on disability in older Italian men and women: rationale, design, and sample characteristics of the Progetto Veneto Anziani (PRO.V.A.) study. J Am Geriatr Soc 2002;50:1535-40.

19 Moderate and vigorous physical activity. Available: https://www.hsph.harvard.edu/ obesity-prevention-source/moderate-and-vigorous-physical-activity/

20 World Health Organization. Physical activity. Available: https://www.who.int/newsroom/fact-sheets/detail/physical-activity\#: :text=Adults\%20aged\%2018\%E2\%80\% 9364\%20years\&text=may\%20increase\%20moderate\%2Dintensity\%20aerobic, week $\% 20$ for\%20additional\%20health\%20benefits

21 Cleveland WS. Robust locally weighted regression and smoothing scatterplots. J Am Stat Assoc 1979;74:829-36.

22 Mok A, Khaw K-T, Luben R, et al. Physical activity trajectories and mortality: population based cohort study. BMJ. 2019:365:12323.

23 Wannamethee SG, Shaper AG. Physical activity in the prevention of cardiovascular disease. Sports Med 2001:31:101-14.

24 Eijsvogels TMH, Thompson PD. Exercise is medicine: at any dose? JAMA 2015:314:1915-6.

25 Cheng S-J, Yu H-K, Chen Y-C, et al. Physical activity and risk of cardiovascular disease among older adults. Int J Gerontol 2013;7:133-6.

26 Parker BA, Kalasky MJ, Proctor DN. Evidence for sex differences in cardiovascular aging and adaptive responses to physical activity. Eur J Appl Physiol 2010:110:235-46.

27 Bennett DA, Du H, Clarke R, et al. Association of physical activity with risk of major cardiovascular diseases in Chinese men and women. JAMA Cardiol 2017:2:1349.

28 Chomistek AK, Cook NR, Rimm EB, et al. Physical activity and incident cardiovascular disease in women: is the relation modified by level of global cardiovascular risk? J Am Heart Assoc 2018;7:e008234.

29 Pinckard K, Baskin KK, Stanford KI. Effects of exercise to improve cardiovascular health. Front Cardiovasc Med 2019;6:69.

30 Lachman S, Boekholdt SM, Luben RN, et al. Impact of physical activity on the risk of cardiovascular disease in middle-aged and older adults: EPIC Norfolk prospective population study. Eur J Prev Cardiol 2018;25:200-8. 